\title{
Nerve growth factor delivery by ultrasound- mediated nanobubble destruction as a treatment for acute spinal cord injury in rats
}

This article was published in the following Dove Press journal:

International Journal of Nanomedicine

2 March 2017

Number of times this article has been viewed

\author{
Zhaojun Song' \\ Zhigang Wang ${ }^{2}$ \\ Jieliang Shen' \\ Shengxi $\mathrm{Xu}{ }^{\prime}$ \\ Zhenming $\mathrm{Hu}^{\prime}$ \\ 'Department of Orthopedics, The \\ First Affiliated Hospital, ${ }^{2}$ Institution \\ of Ultrasound Imaging, The Second \\ Affiliated Hospital, Chongqing Medical \\ University, Chongqing, People's \\ Republic of China
}

Correspondence: Zhenming Hu Department of Orthopedics, The First Affiliated Hospital of Chongqing Medical University, No I Medical College Road, Yuzhong District, 4000I6, Chongqing, People's Republic of China

Email spinecenterhu@163.com
Background: Spinal cord injuries (SCIs) can cause severe disability or death. Treatment options include surgical intervention, drug therapy, and stem cell transplantation. However, the efficacy of these methods for functional recovery remains unsatisfactory.

Purpose: This study was conducted to explore the effect of ultrasound (US)-mediated destruction of poly(lactic-co-glycolic acid) (PLGA) nanobubbles (NBs) expressing nerve growth factor (NGF) (NGF/PLGA NBs) on nerve regeneration in rats following SCI.

Materials and methods: Adult male Sprague Dawley rats were randomly divided into four treatment groups after Allen hit models of SCI were established. The groups were normal saline (NS) group, $N G F$ and NBs group, $N G F$ and US group, and $N G F /$ PLGA NBs and US group. Histological changes after SCI were observed by hematoxylin and eosin staining. Neuron viability was determined by Nissl staining. Terminal deoxynucleotidyl transferase-mediated dUTP-biotin nick end labeling staining was used to examine cell apoptosis. NGF gene and protein expressions were detected by quantitative reverse transcription polymerase chain reaction and Western blotting. Green fluorescent protein expression in the spinal cord was examined using an inverted fluorescence microscope. The recovery of neural function was determined using the Basso, Beattie, and Bresnahan test.

Results: $N G F$ therapy using US-mediated $N G F /$ PLGA NBs destruction significantly increased NGF expression, attenuated histological injury, decreased neuron loss, inhibited neuronal apoptosis in injured spinal cords, and increased BBB scores in rats with SCI.

Conclusion: US-mediated $N G F /$ PLGA NBs destruction effectively transfects the $N G F$ gene into target tissues and has a significant effect on the injured spinal cord. The combination of US irradiation and gene therapy through NGF/PLGA NBs holds great promise for the future of nanomedicine and the development of noninvasive treatment options for SCI and other diseases.

Keywords: nanobubbles, nerve growth factor, spinal cord injury, apoptosis, gene therapy

\section{Introduction}

Spinal cord injuries (SCIs) can cause severe disability or death. The most common causes of SCI are motor vehicle accidents (38\%), falls (22\%), violence (13.5\%), and sport/recreational accidents $(9 \%) .{ }^{1}$ In SCI, structural and functional damage of the spinal cord occurs by primary and secondary injury. This results in loss of movement and sensation and sphincter and autonomic nerve dysfunction below the damaged plane. These effects can damage the patient's mental health and places an immense burden on society from a public health perspective. ${ }^{2-4}$

The principal treatments for SCI include surgical stabilization and decompression. Physiotherapy and neurotrophic rehabilitation attenuate further damage, relieve spinal 
cord ischemia and hypoxia, save the damaged area, rescue necrotic neurons, and promote neuronal function. ${ }^{5-8}$ In recent years, gene therapy has provided an exciting new direction for research, and substantial progress has been made with improved vectors. Viral vectors have a high transfection efficiency, but their application is restricted because they are cytotoxic with high immunogenicity. ${ }^{9,10}$ Compared with viral vectors, nonviral vectors such as liposomes and polymeric nanospheres are safe and less toxic. However, their utilization is limited by instability and inefficiency. ${ }^{11-13}$ Therefore, the search for a safe, efficient, and highly specific transfection system for gene therapy of SCI has become a focus of interest for researchers. Poly(lactic-co-glycolic acid) (PLGA) nanobubbles (NBs) are nonviral vectors that possess unique advantages as gene carriers, such as targeting, slow release, and penetration. ${ }^{14-16}$ These properties make PLGA NBs ideal candidates for use as drug or gene carriers for the treatment of SCI.

Recently, there has been a rapid development in ultrasound (US)-mediated microbubble destruction (UTMD). UTMD improves the transfection efficiency of exogenous genes noninvasively by sonoporation of microbubbles via US irradiation. Many studies have shown that UTMD is a safe and effective method for transfection. ${ }^{17,18}$ To date, UTMD has been successfully used for gene transfection in diseases of the heart, lung, brain, uterus, pancreas, kidneys, retina, skin, and testes. ${ }^{19-27}$ To the best of our knowledge, very few studies have investigated the use of US-mediated gene therapy for SCI. Thus, it is still unknown whether USmediated gene transfection represents an effective treatment strategy for SCI.

Nerve growth factor (NGF) is one of the best-studied neurotrophic factors (NTFs) and has been shown to promote the growth, differentiation, survival, and synaptogenesis of central and peripheral neurons. ${ }^{28}$ In this study, we performed US-mediated destruction of $N G F /$ PLGA NBs and investigated its ability to promote the growth, differentiation, and synaptogenesis of neurons for repair of damaged spinal cord tissues in rats after SCI.

\section{Materials and methods}

\section{Sample preparation and assay kits}

PLGA $(50: 50$, molecular weight $[\mathrm{MW}]=12,000)$ and polyvinyl alcohol (PVA, MW =25,000) were purchased from Daigang Biomaterial Co., Ltd. The pCMV6-NGF-GFP plasmid was purchased from OriGene Technologies, Inc. and extracted using the E.Z.N.A. Plasmid Maxi Kit (D6942-01; Qingdao Superri Bio-equip Co., Ltd.). Primary anti-NGF antibody and secondary antibodies were purchased from ABclonal Biotechnology Co., Ltd. Real-time quantitative reverse transcription polymerase chain reaction (qRT-PCR) kits were purchased from Takara Bio Inc. (RR047A). Terminal deoxynucleotidyl transferase-mediated dUTPbiotin nick end labeling (TUNEL) Apoptosis Assay Kit was purchased from Roche (Roche Applied Science).

\section{Preparation of NGF/PLGA NBs}

$N G F /$ PLGA NBs were fabricated using a double-emulsion process as reported previously. ${ }^{29}$ First, $1 \mathrm{mg} / \mathrm{mL}$ pCMV6$N G F-G F P$ plasmid was added to the organic phase of $2 \mathrm{~mL}$ $\mathrm{CH}_{2} \mathrm{Cl}_{2}$ containing $25 \mathrm{mg}$ dissolved PLGA. This mixture was emulsified using an ultrasonic processor at $100 \mathrm{~W}$ for $60 \mathrm{~s}$ in an ice bath. Subsequently, $10 \mathrm{~mL}$ cold PVA solution $(1 \% \mathrm{w} / \mathrm{v})$ was poured into this initial emulsion, and the mixture was emulsified using an ultrasonic processor for $3 \mathrm{~min}$ at $75 \mathrm{~W}$ in an ice bath. The final solution was stirred for $3 \mathrm{~h}$ by a magnetic stirrer and centrifuged at 13,000× $g$ for $5 \mathrm{~min}$. The supernatant was discarded, and the precipitate was washed in deionized water. Centrifugation and washing were repeated three times. Finally, the washed precipitate was lyophilized for $48 \mathrm{~h}$. The dried samples were harvested, filled with perfluorocarbon gas, and stored at $-20^{\circ} \mathrm{C}$ until further use.

The mean diameter, polydispersity index (PDI), and zeta potential of the samples were analyzed by a Laser Particle Size Analyzer System (Zeta SIZER, Malvern). The morphological characterization of the NBs was performed using a light microscope (Olympus CKX41) and a scanning electron microscope (SEM; Hitachi S-340 0N). Transmission electron microscopy (TEM; Hitachi H-760 0 ) was used to estimate the shape of the nanoparticles. The gene encapsulation efficiency was determined using an ultraviolet spectrophotometry method (Thermo NanoDrop 2000; Thermo Fisher Scientific). The following equation was used to calculate the gene encapsulation efficiency: Encapsulation efficiency $=\left(\mathrm{W}_{1} / \mathrm{W}_{2} \times 100\right) \%$, where $\mathrm{W}_{1}$ represents the gene amount in $N G F /$ PLGA NBs and $\mathrm{W}_{2}$ represents the total amount of NGF used to prepare the $N G F /$ PLGA NBs. ${ }^{29,30}$ Each experiment was performed in triplicate. We compared $N G F$ encapsulation efficiency of five different $N G F$ plasmid concentrations $(0.1,0.2$, $0.4,0.8$, and $1 \mathrm{mg}$ ). In vitro sonication was performed to assess gene release behavior using a gene transfection instrument (Model UTG 1025; Institution of Ultrasound Imaging, Chongqing Medical University, Chongqing). Sonication parameters were set at $1.5 \mathrm{~W}$ for $60 \mathrm{~s}$. The $N G F /$ 
PLGA NBs in $10 \mathrm{~mL}$ phosphate-buffered saline (PBS) were transferred to dialysis bags (molecular weight cutoff: $10,000 \mathrm{Da}$ ) and placed in a reservoir of $100 \mathrm{~mL}$ PBS after insonation. At appropriate intervals, $1 \mathrm{~mL}$ of the dialysate was extracted and used to determine the gene concentration by ultraviolet spectrophotometry. Consecutively, $1 \mathrm{~mL}$ of fresh PBS was added back to the reservoir to maintain a constant volume. The accumulative ratios of the released NGF were calculated.

\section{Experimental animal models and drug injection}

This study was conducted in strict accordance with the National Institutes of Health Guide for the Care and Use of Laboratory Animals, and all the experimental protocols were approved by the animal ethics committee of Chongqing Medical University.

A modified Allen method was used to induce contusion injury. ${ }^{31}$ The tenth segment of the thoracic cord was exposed and injured by dropping a $10 \mathrm{~g}$ rod from a distance of $5 \mathrm{~cm}$ on to the spinal cord and letting the rod rest for $3 \mathrm{~min}$ on the lesion site. Adult male Sprague Dawley rats weighing 180-200 g were randomly separated into the following four groups and administered the following treatments:

1. Control group: $0.5 \mathrm{~mL}$ normal saline (NS) was injected via the tail vein $(n=37)$.

2. $N G F+$ NBs group: $0.5 \mathrm{~mL} N G F$ plasmid $(50 \mu \mathrm{g})$ and pure PLGA NBs solution were injected via the tail vein $(\mathrm{n}=37)$.

3. $N G F+$ US group: $0.5 \mathrm{~mL} N G F$ plasmid $(50 \mu \mathrm{g})$ solution was injected via the tail vein and then subjected to US irradiation for $5 \mathrm{~min}(\mathrm{n}=37)$.

4. $N G F /$ PLGA NBs + US group: $0.5 \mathrm{~mL} \mathrm{NGF}(50 \mu \mathrm{g}) /$ PLGA NBs solution was injected via the tail vein and then subjected to US irradiation for $5 \min (n=37)$.

All rats received the corresponding drug injections every $12 \mathrm{~h}$ and were then subjected to US irradiation $\left(2.5 \mathrm{~W} / \mathrm{cm}^{2}\right)$ for $5 \mathrm{~min}$. This was continued for 3 days, and then specimens were collected. All surgeries were performed under chloral hydrate anesthesia $(10 \%, 0.3 \mathrm{~mL} / 100 \mathrm{~g})$, and all efforts were made to minimize animal suffering.

Following surgery, all rats were housed in separate cages to prevent them from attacking each other. Food and water were provided ad libitum. Intraperitoneal injections of penicillin (2,000 U/100 g) were administered twice a day for 3 days. Urination was stimulated in rats by massaging the bladder twice a day for 7 days. Dressings were kept dry and replaced every 3 days.

\section{qRT-PCR}

Total RNA was isolated from the spinal cord tissue using Trizol reagent (ComWin Biotech Co., Ltd., Beijing, China). RNA was reverse transcribed to complementary DNA (cDNA) using the PrimeScript ${ }^{\mathrm{TM}}$ RT reagent kit (Perfect Real Time kit) (TAKARA BIO INC). The cDNA samples were prepared for qRT-PCR using a SYBR Premix Ex Taq ${ }^{\mathrm{TM}}$ (Perfect Real Time kit) (TAKARA BIO INC). The $N G F$ primer sequences were 5'-GTTTAGCACCCAGCCTCC-3' (sense) and 5'-GGTCTTATCTCCAACCCACA-3' (antisense), and $G A P D H$ (internal control) primer sequences were 5'-GGGGTGATGCTGGTGCTGAGTATG-3' (sense) and 5'-CCGCCTGCTTCACCACCTTCTT-3' (antisense). qRT-PCR was carried out in 96-well plates using an ABI 7500 Real-Time PCR System and 7500 System Software (Applied Biosystems, Alameda, CA, USA). Each qRT-PCR analysis was performed in triplicate. qRT-PCR data were analyzed by the $2^{-\Delta \Delta C \mathrm{CT}}$ method. ${ }^{32}$

\section{Western blotting}

Tissue lysates were prepared from samples frozen at $-80^{\circ} \mathrm{C}$. Samples were homogenized in RIPA lysis buffer to obtain protein lysates. The lysates were centrifuged at $10,000 \times g$ for $10 \mathrm{~min}$ at $4^{\circ} \mathrm{C}$, and the supernatants were transferred into separate tubes. Protein concentrations were determined using the bicinchoninic acid assay (Beyotime Institute of Biotechnology, China). Equal amounts of protein were separated by electrophoresis on 5\% sodium dodecyl sulfate polyacrylamide gels (SDS-PAGE) and then transferred on to polyvinylidene fluoride (PVDF) membranes. The membranes were incubated with a mouse anti-rat NGF monoclonal antibody at $4{ }^{\circ} \mathrm{C}$ in $5 \%$ skim milk overnight. Then, membranes were incubated with a goat anti-mouse secondary immunoglobulin $\mathrm{G}$ ( $\mathrm{IgG}$ ) conjugated to horseradish peroxidase. Labeled protein bands were visualized using an electrochemiluminescence (ECL) Western blot substrate kit (Millipore, USA). NGF protein bands were normalized to $\beta$-actin, and all immunoblots were quantified with Image J software.

\section{Green fluorescent protein (GFP) quantification by inverted fluorescence microscopy}

To monitor the location and expression of $N G F$, a plasmid DNA encoding a GFP reporter gene was introduced using optimal transfection conditions. Spinal cord specimens were cut into frozen sections $(10 \mu \mathrm{m})$, and GFP was detected by an inverted fluorescence microscope (Leica DMI4000B, 
Germany). Semiquantitative analysis of GFP fluorescence intensity was performed using ImageJ software.

\section{Hematoxylin and eosin (HE) and Nissl staining}

Spinal cord tissues were immersed in $4 \%$ paraformaldehyde for $24 \mathrm{~h}$, transferred to $70 \%$ ethanol, dehydrated through a serial alcohol gradient, embedded in paraffin wax blocks, and serially sectioned into $8 \mu \mathrm{m}$-thick segments. Tissue sections were dewaxed in xylene, rehydrated through decreasing concentrations of ethanol, and washed in distilled water for $\mathrm{HE}$ and Nissl staining. Stained cells were observed using a light microscope (Leica DM500 ICC50).

\section{Detection of apoptotic cells by TUNEL assay}

Neuronal apoptosis in the injured region of the spinal cord was analyzed using a TUNEL staining kit (Roche) according to the manufacturer's instructions. Briefly, the tissue sections were dewaxed in xylene, rehydrated through decreasing concentrations of ethanol, washed in distilled water, treated with proteases, and incubated with the TUNEL reaction mixture. The nuclei were counterstained with 4',6-diamidino2-phenylindole (DAPI) (Roche). TUNEL-positive cells had a pyknotic nucleus with green fluorescence, indicative of apoptosis. Sections were imaged using a fluorescence microscope (Leica DMI4000B). The TUNEL reaction was also visualized by chromogenic staining with diaminobenzidine (DAB) (Roche). TUNEL-positive cells were stained brownish-yellow, and positive cells were counted. Sections were imaged using a microscope (Leica DM500 ICC50).

\section{Basso, Beattie, and Bresnahan (BBB) locomotor rating scale}

The recovery of neural function was assessed 7, 14, 21, and 28 days after SCI using the BBB locomotor rating scale. ${ }^{33}$ Rats were placed in an open field $(80 \times 130 \times 30 \mathrm{~cm})$ with a pasteboard-covered nonslippery floor. In each testing session, individual rats were observed for $3 \mathrm{~min}$.

\section{Statistical analysis}

SPSS18.0 software (IBM Corporation, Armonk, NY, USA) was used for statistical analysis. All data were recorded as mean values \pm standard deviation (SD). One-way analysis of variance was used to determine the statistical differences within groups at multiple time points. Student's $t$-test was used to analyze differences between two groups at the same time point. A $P$-value of $<0.05$ was considered statistically significant.

\section{Results}

\section{Characterization of NGF/PLGA NBs}

The mean diameter of $N G F /$ PLGA NBs was $215.3 \pm 55.29 \mathrm{~nm}$. The PDI was 0.027 (Figure 1A), and the zeta potential was $-11.3 \pm 5.65 \mathrm{mV}$ (Figure 1B). SEM analysis revealed that $N G F /$ PLGA NBs were highly dispersed and had a well-defined spherical morphology (Figure 1C). The plasmid solution was observed in the core of NBs by TEM (Figure 1D). The encapsulation efficiency of $N G F /$ PLGA NBs was measured by the ultraviolet spectrophotometry method and was highest $(34.33 \% \pm 2.02 \%)$ when $0.4 \mathrm{mg}$ of $N G F$ DNA plasmid was used (Figure 1E). The release efficiency of $N G F$ from $N G F /$ PLGA NBs, from the accumulative ratios, was measured by ultraviolet spectrophotometry after low-frequency ultrasonic irradiation. Within $12 \mathrm{~h}$ after ultrasonic irradiation, 52.7\% of $N G F$ was released from $N G F /$ PLGA NBs and $62.6 \%$ of $N G F$ was released after $48 \mathrm{~h}$ (Figure 1F).

\section{Verification of the animal model}

A modified Allen method was used to induce contusion injury at the T10 segments (Figure 2A and B). Randomly selected rats from each group were analyzed by 7.0 $\mathrm{T}$ magnetic resonance imaging (MRI, Biospec 70/20USR; BRUKER, Germany) (Figure 2C and D), and specimens were collected for HE staining 1 day after surgery (Figure 2E). Pathological analysis verified the animal model, and 7.0 T MRI confirmed that SCI was accurately induced.

\section{Total NGF expression in vivo after transfection}

NGF protein and mRNA expression were measured 7 and 14 days after SCI. The qRT-PCR data were analyzed by the $2^{-\triangle \triangle C T}$ method. $N G F$ mRNA expression was significantly higher in the $N G F / \mathrm{PLGA} \mathrm{NBs}+\mathrm{US}$ group compared with other groups at 7 and 14 days after transfection $(P<0.05$; Figure $3 \mathrm{~A}$ and $\mathrm{B})$. Furthermore, expression was significantly different $(P<0.05)$ between all pairs of groups, except between NS and $N G F+\mathrm{NBs} . N G F$ mRNA expression was highest in the $N G F /$ PLGA NBs + US than all other groups (Figure 3A and B).

NGF protein expression was detected by Western blotting. A single band was observed for each group. NGF protein expression was highest in the $N G F / \mathrm{PLGANBs}+\mathrm{US}$ group 7 and 14 days after treatment, compared with the other groups $(P<0.05$; Figure 3C-F). NGF protein expression was higher in the $N G F+$ US group than the NS and the $N G F+$ NBs groups $(P<0.05)$ in the early stages after treatment. This indicates that US irradiation alone can improve the gene transfection efficiency. 


\section{A}

PDI: 0.027

Intercept: 0.953

Result quality Good

$\begin{array}{llll} & \begin{array}{l}\text { Size } \\ (\mathbf{d} \cdot \mathbf{n m})\end{array} & \begin{array}{l}\% \\ \text { intensity: }\end{array} & \begin{array}{l}\text { SD } \\ (\mathbf{d} \cdot \mathbf{n m})\end{array} \\ \text { Peak 1: } & 226.7 & 100.0 & 55.29 \\ \text { Peak 2: } & 0.000 & 0.0 & 0.000 \\ \text { Peak 3: } & 0.000 & 0.0 & 0.000\end{array}$

Size distribution by intensity

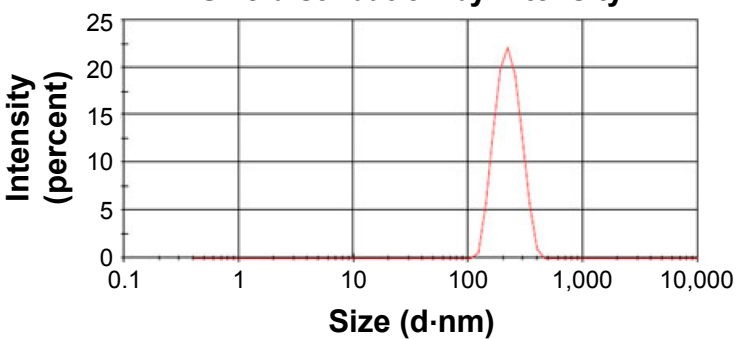

C

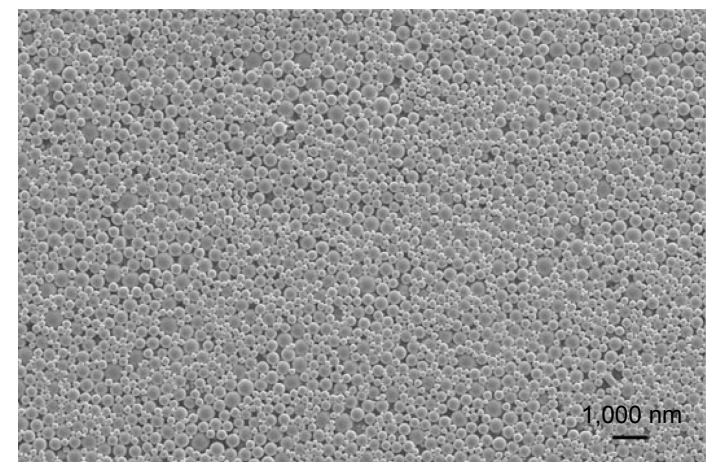

E

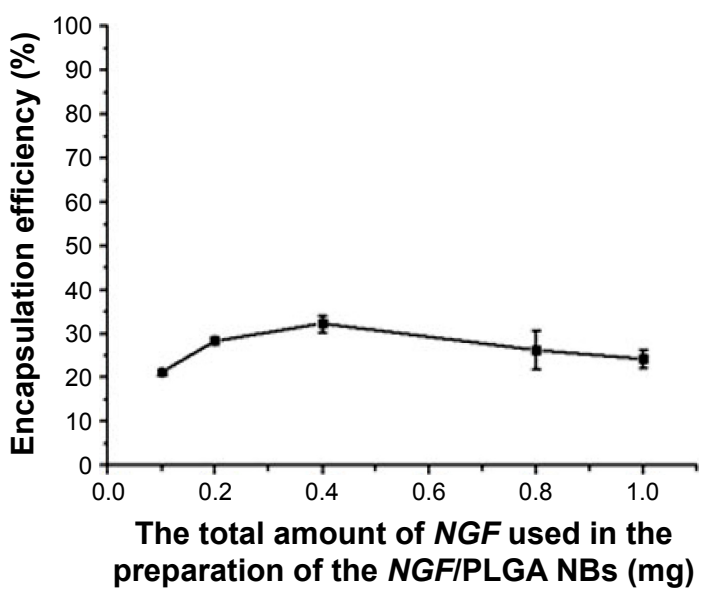

B

Mean Area SD

$(\mathrm{mV}) \quad(\%) \quad(\mathrm{mV})$

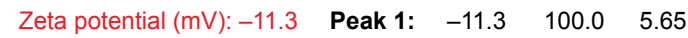

Zeta deviation (mV): $5.65 \quad$ Peak 2: $\quad \begin{array}{lllll}0.00 & 0.0 & 0.00\end{array}$

Conductivity $(\mathrm{mS} / \mathrm{cm}): 0.0157$ Peak 3: $\quad \begin{array}{llll}0.00 & 0.0 & 0.00\end{array}$

Result quality Good

Zeta potential distribution

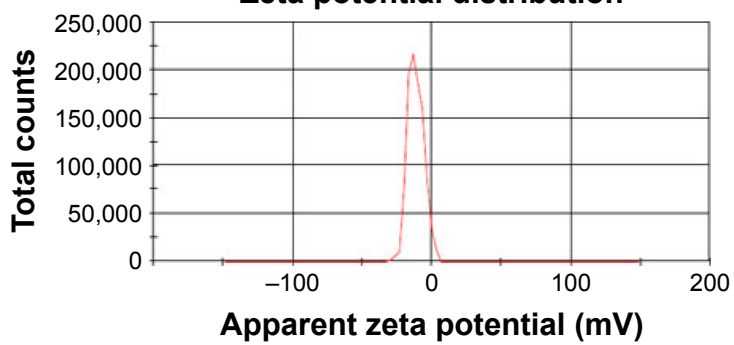

D

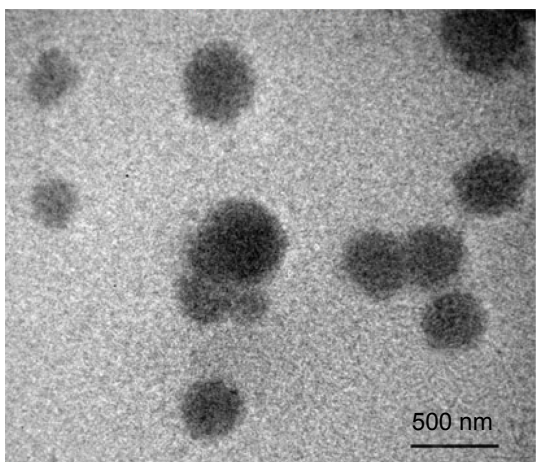

$\mathbf{F}$

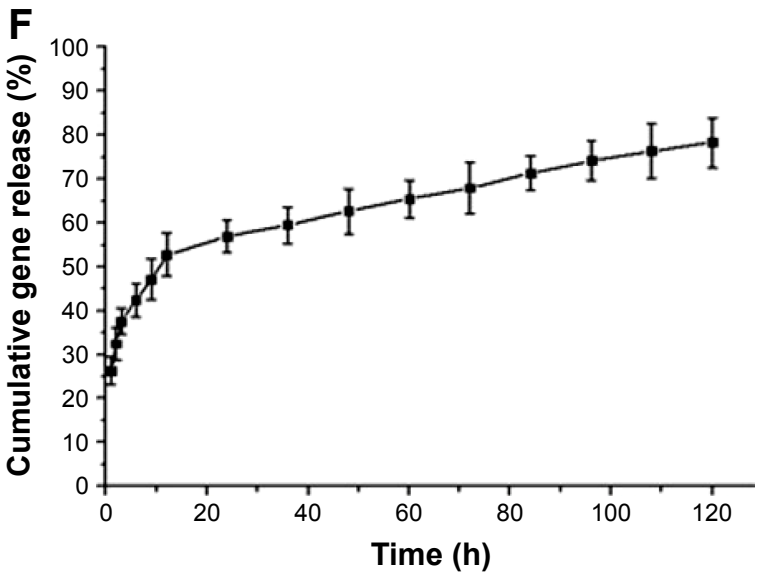

Figure I NGF/PLGA NBs are highly dispersed and have a well-defined spherical morphology.

Notes: (A) NGF/PLGA NBs are well dispersed (PDI =0.027) and small, with a narrow size distribution (215.3 $\pm 55.29 \mathrm{~nm})$. (B) Zeta potential by Laser Particle Size Analyzer System showing that NGF/PLGA NBs have good stability. The SEM image $(\mathbf{C}, \times 1,000)$ and TEM image $(\mathbf{D}, \times 2,500)$ show that NGF/PLGA NBs are highly dispersed with a well-defined spherical morphology. (E) The encapsulation efficiency was highest (34.3\%) when $0.4 \mathrm{mg}$ NGF DNA plasmid was used. (F) Within I2 h of ultrasonic irradiation, $52.7 \%$ of NGF was released from the NGF/PLGA NBs and $62.6 \%$ of NGF was released after $48 \mathrm{~h}$.

Abbreviations: NGF, nerve growth factor; PLGA, poly(lactic-co-glycolic acid); NBs, nanobubbles; PDI, polydispersity index; SEM, scanning electron microscope; TEM, transmission electron microscopy; SD, standard deviation.

\section{GFP expression}

GFP expression was detected by fluorescence microscopy 7 and 14 days after transfection. GFP expression was higher in the $N G F /$ PLGA NBs + US group than other groups after transfection $(P<0.05$; Figure $4 \mathrm{~A}$ and $\mathrm{B})$. The intensity of green fluorescence was highest in the $N G F /$ PLGA NBs + US group, second highest in the $N G F+$ US group, followed by the $N G F$ group and NS group. GFP expression differed significantly $(P<0.05)$ between all pairs of groups, except between the NS group and the NGF group. GFP expression 

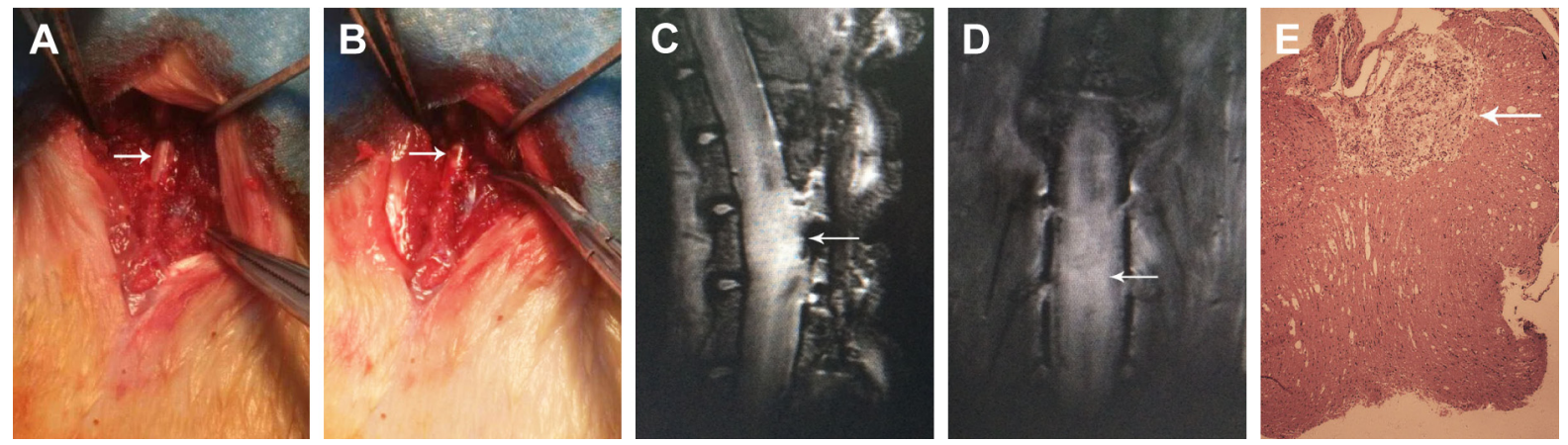

Figure $2 \mathrm{MRI}$ image analysis and pathological evaluation indicate the success of the $\mathrm{SCl}$ model.

Notes: (A) Exposed rat spinal cord (arrow). (B) The spinal cord was surgically exposed and hit with $10 \mathrm{~g}$ rod, leaving obvious contusions (arrow). (C) and (D) MR image of rat spinal cord shows contusion injury at the TIO segment. Sagittal image (C) and coronal image (D) show long T2 signals in the SCl site (arrows). (E) HE staining showing hemorrhage, disordered white matter nerve fibers, and formation of cavities in the $\mathrm{SCl}$ site (arrow).

Abbreviations: MRI, magnetic resonance imaging; $\mathrm{SCl}$, spinal cord injury; $\mathrm{HE}$, hematoxylin and eosin.

A

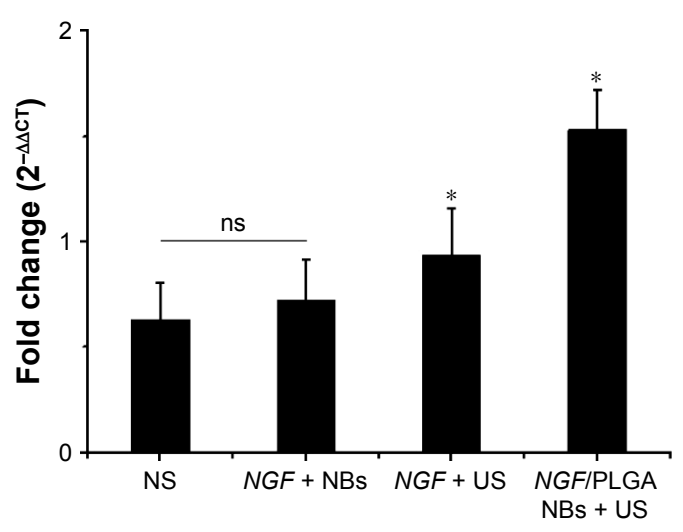

C

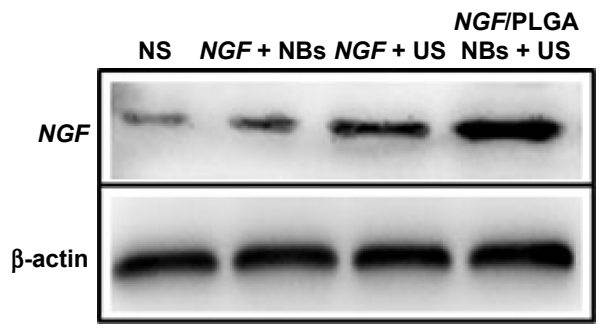

E

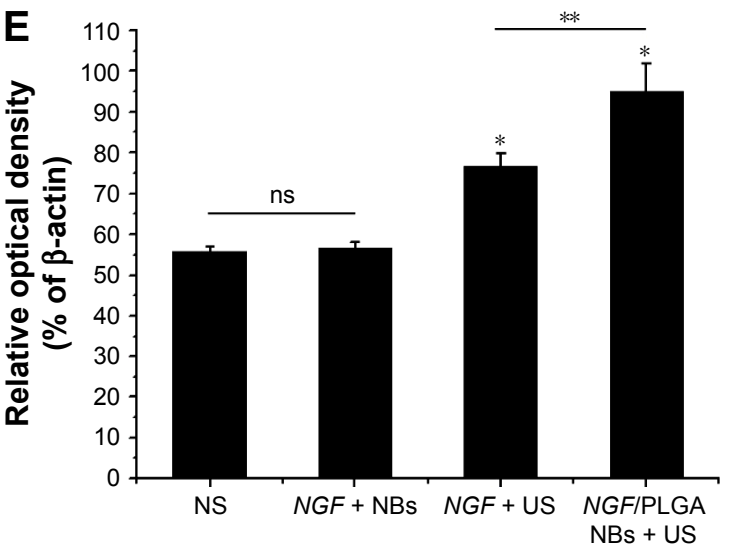

B

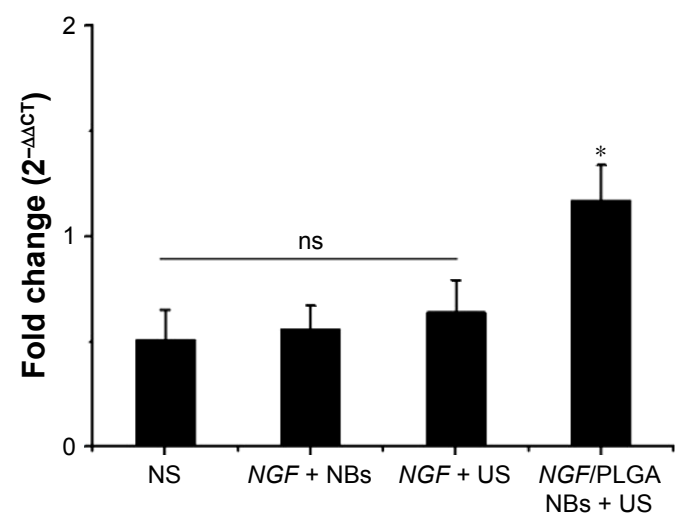

D
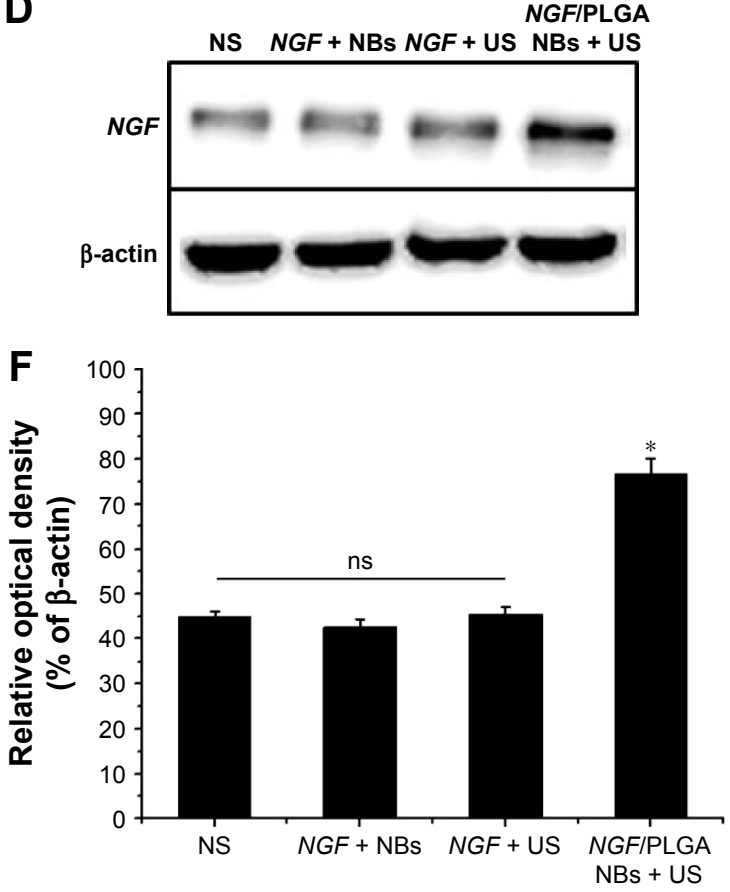

Figure 3 NGF therapy using US-mediated NGF/PLGA NBs destruction increases NGF expression after in vivo transfection.

Notes: Graphs showing the fold-change increase in NGF expression in the NGF/PLGA NBs + US group by qRT-PCR at 7 days (A) and I4 days (B) after transfection. NGF protein expression was higher after US-mediated NB destruction and NGF therapy at 7 days (C and E) and 14 days (D and $\mathbf{F})$. $* P<0.05$ compared with other groups. $* * P<0.05$ compared with NGF + US group.

Abbreviations: NS, normal saline; NGF, nerve growth factor; US, ultrasound; PLGA, poly(lactic-co-glycolic acid); NBs, nanobubbles; qRT-PCR, quantitative reverse transfection polymerase chain reaction; ns, not significant. 
A

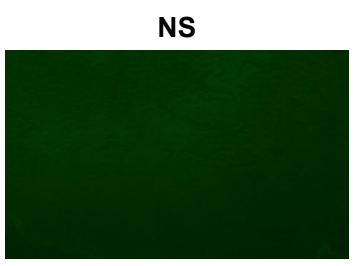

B

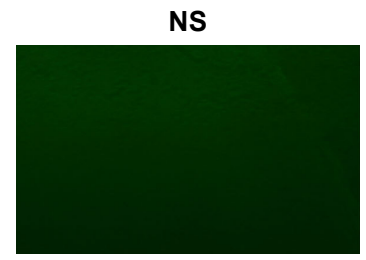

7 days
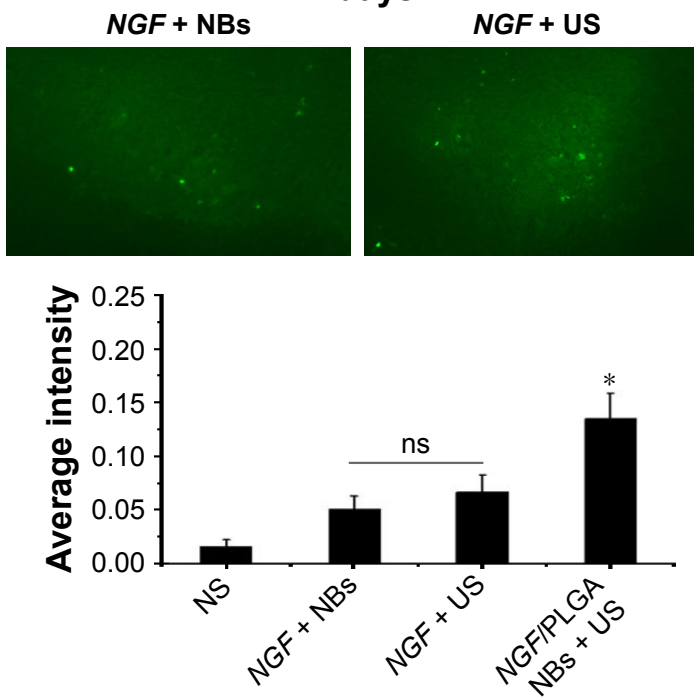

14 days
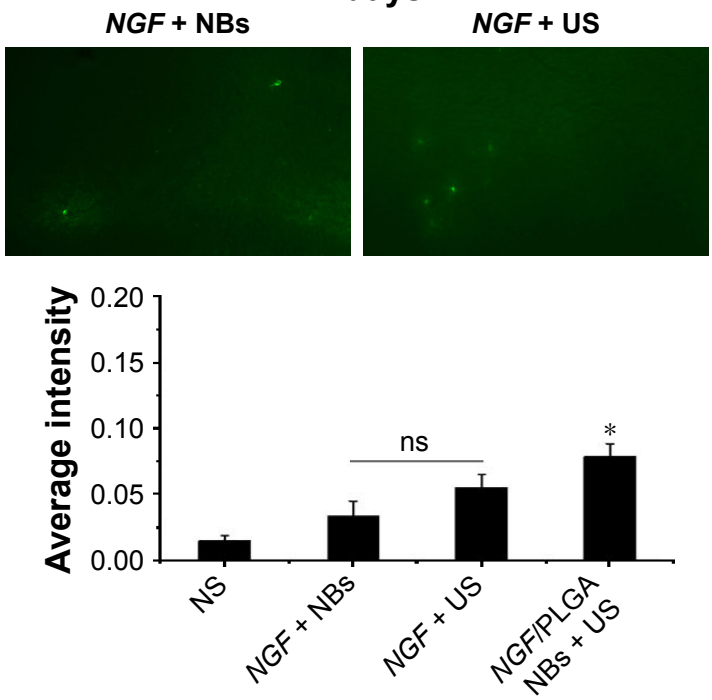

NGF/PLGA NBs + US

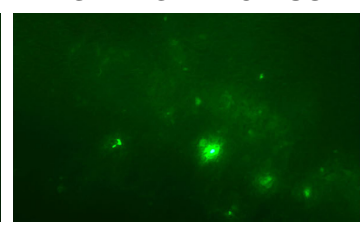

NGF/PLGA NBs + US

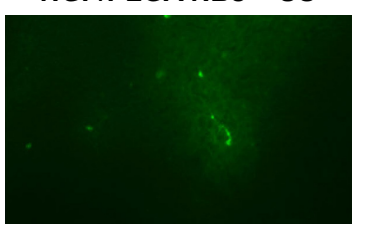

Figure 4 NGF therapy using US-mediated NGF/PLGA NBs destruction increased GFP expression.

Notes: (A) GFP expression 7 days after SCl $(\times 200)$. GFP expression was the strongest in NGF/PLGA NBs + US group, second strongest in NGF + US group, followed by the NGF group, and no expression was found in NS group. (B) GFP expression 14 days after $\mathrm{SCl}(\times 200)$. GFP expression was highest in the NGF/PLGA NBs + US group. $* P<0.05$, compared with the other three groups.

Abbreviations: NGF, nerve growth factor; US, ultrasound; PLGA, poly(lactic-co-glycolic acid); NBs, nanobubbles; GFP, green fluorescent protein; SCl, spinal cord injury; NS, normal saline; ns, not significant.

was highest in the $N G F /$ PLGA NBs + US group at 7 and 14 days after transfection.

\section{General histopathology and Nissl staining}

Histopathological alterations caused by SCI were examined using HE staining 7 and 14 days after treatment. Hemorrhage was observed in the gray matter, and only a small number of neurons survived. Swollen axons and many vacuoles were visible in the remaining white matter in all groups after SCI. The extent of histopathological changes in the $N G F /$ PLGA $\mathrm{NBs}+$ US group was attenuated compared with other groups, at day 7 and day 14 . Some glial cell proliferation was detected and many neurons survived and showed normal morphology (Figure 5).

Nissl staining was used to verify neuron viability. Analysis of variance revealed a statistically significant difference in the number of viable neurons among the four groups 7 and 14 days after transfection. Furthermore, significantly fewer Nissl bodies were detected in the NS group and the $N G F+$ NBs group than other groups $(P<0.05)$, while significantly more Nissl bodies were observed in the $N G F /$ PLGA NBs + US group compared with other groups $(P<0.01)$. In addition, Nissl bodies in the $N G F /$ PLGA NBs + US group were large and plump (Figure 6). 
A

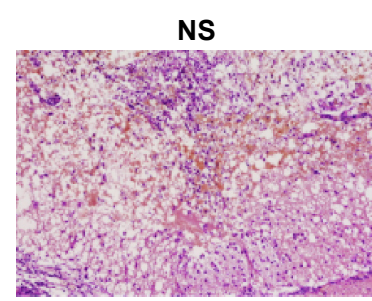

B

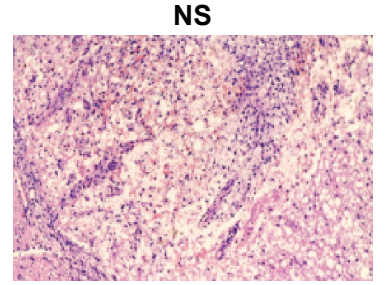

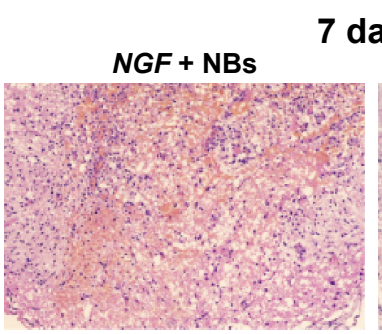

7 days

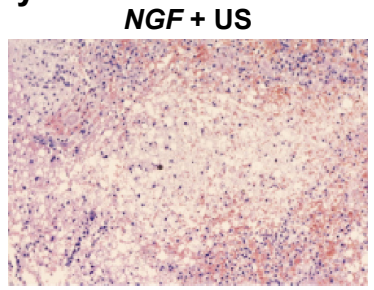

14 days
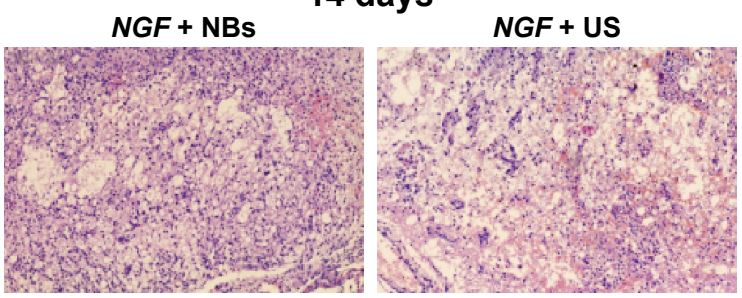

NGFIPLGA NBs + US

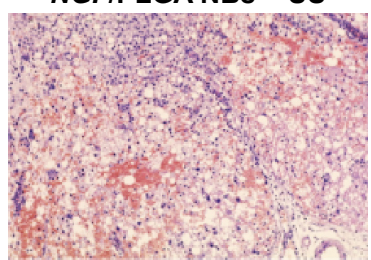

NGFIPLGA NBs + US

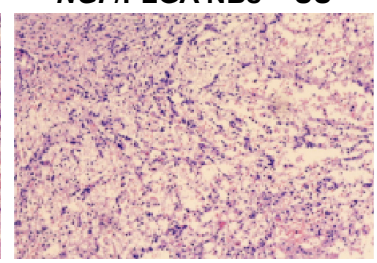

Figure 5 NGF therapy using US-mediated NGF/PLGA NBs destruction attenuates histological injury.

Notes: (A) General histopathology of the spinal cord 7 days after $\mathrm{SCl}(\times 200)$. Hemorrhage was observed in the gray matter area and only a small number of neurons survived in all groups. However, the pathological changes were less severe in the NGF/PLGA NBs + US group. (B) General histopathology of the spinal cord I4 days after $\mathrm{SCl}(\times 200)$. After NGF therapy through US-mediated NB destruction, more neurons survived and showed normal morphology. Mild glial proliferation was detected in the NGF/PLGA NBs + US group.

Abbreviations: NGF, nerve growth factor; US, ultrasound; PLGA, poly(lactic-co-glycolic acid); NBs, nanobubbles; SCl, spinal cord injury; NS, normal saline.

\section{TUNEL assay}

Neuronal apoptosis was measured in the injured region of spinal cord using a TUNEL assay 14 days after transfection. The TUNEL-DAPI assay showed reduced apoptosis in the $N G F /$ PLGA NBs + US group (Figure 7A). There were many TUNEL-positive cells in the spinal cord of rats in the NS group and $N G F+$ NBs group. The number of TUNELpositive cells decreased slightly in the US $+N G F$ group. These findings indicate that US-mediated NB destruction combined with $N G F$ treatment significantly reduced the number of apoptotic cells $(P<0.01$; Figure 7B). This suggests that $N G F$ therapy through US-mediated NB destruction protects neurons effectively by inhibiting apoptosis.

\section{BBB locomotor rating scale}

Recovery of neural function in the hindlimb was assessed $7,14,21$, and 28 days after treatment. All rats had normal limb function and a BBB score of 21 before SCI. Immediately after the operation, the BBB score reduced to 0 in all rats, indicating that an SCI model was effectively established. BBB scores were significantly higher following 7 days of $N G F /$ PLGA $\mathrm{NBs}+\mathrm{US}$ treatment. Recovery continued to improve more in rats treated with $N G F /$ PLGA NBs + US compared with other groups, as shown by higher BBB scores $(P<0.05$; Figure 8$)$.

\section{Discussion}

The clinical treatment of SCI poses difficult problems. In $\mathrm{SCI}$, the force of the primary and secondary injury destroys neural tissues, which may lead to permanent loss of function. When the spinal cord is injured, cells and molecules associated with scar formation are activated to form a glial scar, which can limit axon regeneration. Although many promising molecular strategies have emerged to reduce secondary injury and promote axonal regrowth, there is still no effective cure and functional recovery remains limited. ${ }^{34}$ In recent years, the use of therapeutic exogenous genes to promote neuronal regeneration has gained much interest, and clinical trials have been initiated. ${ }^{28,35}$ Nevertheless, the safety and continuous expression of these exogenous genes must be addressed before they can be implemented in clinical practice.

NGF is one of the best studied NTFs. Previous studies have shown that NGF protects neurons from damage and promotes regeneration in the central nervous system. ${ }^{28}$ The purpose of gene therapy is to rectify abnormal protein expression at the genetic level. Introducing exogenous $N G F$ to correct decreased NGF levels represents a promising treatment option for central nervous system disorders. The mechanism behind the NTFlike activity of NGF is well understood; however, its therapeutic efficacy is not satisfactory. This may be attributed to the blood-brain barrier, which prevents most drugs or genes from entering the brain. ${ }^{36,37}$ Several studies have demonstrated that low-power focused US irradiation following the intravenous administration of an US contrast agent can be used to induce reproducible and consistent focal opening of the blood-brain barrier without any permanent damage to brain tissue. ${ }^{38-41}$ UTMD facilitates the transfer of extracellular molecules 
A
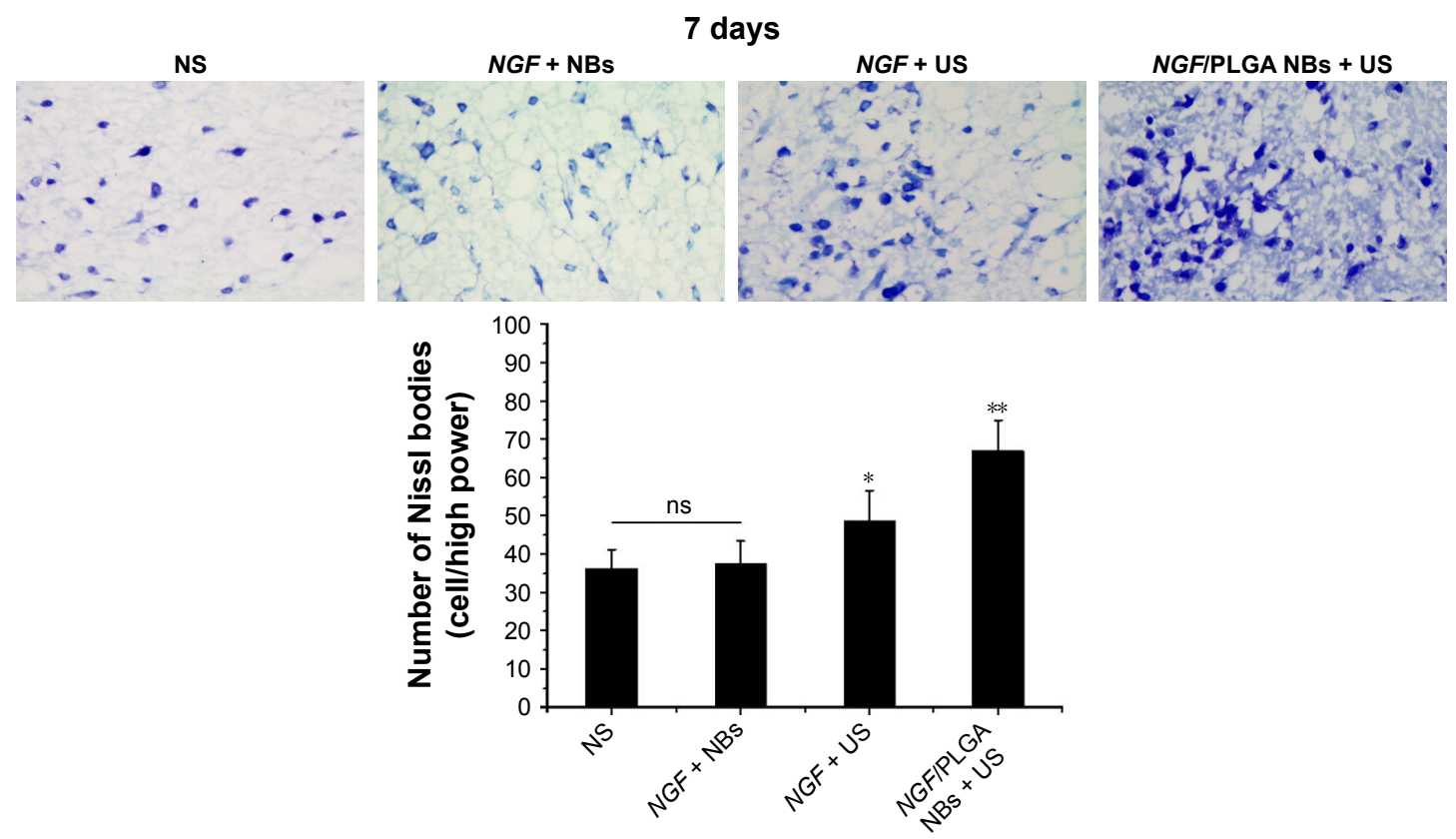

B
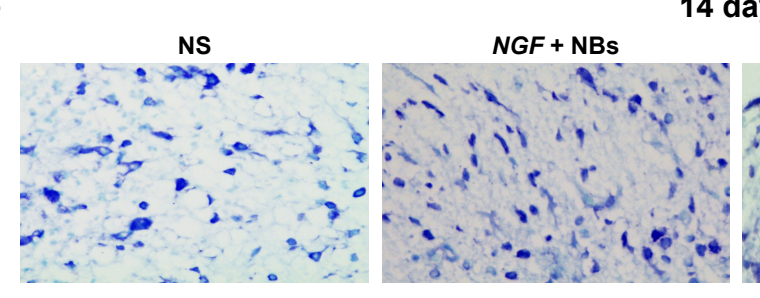

\section{days}
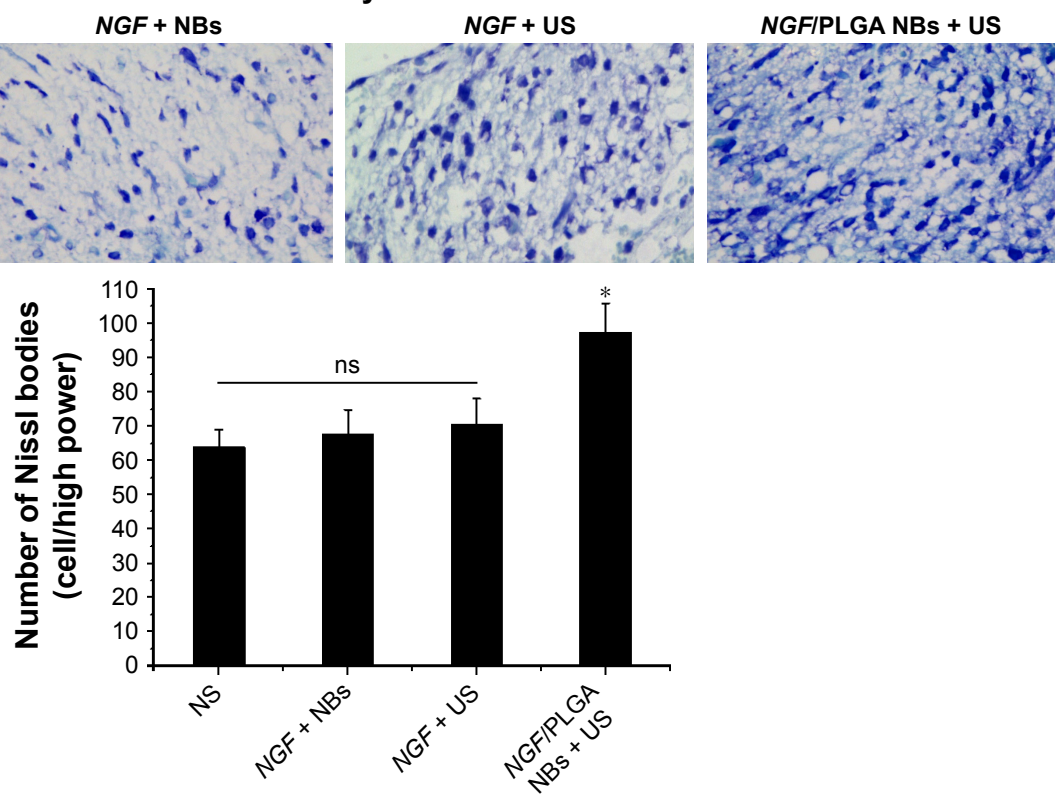

Figure 6 NGF therapy using US-mediated NGF/PLGA NBs destruction decreases neuron loss.

Notes: Nissl staining of neurons in different groups at 7 days $(\mathbf{A}, \times 400)$ and 14 days $(\mathbf{B}, \times 400)$ after treatment. Neurons in the NS group and the NGF + US group showed lighter staining. More Nissl bodies were detected in the NGF/PLGA NBs + US group, and these Nissl bodies were large and plump. $* P<0.05$, compared with other groups. $* * P<0.05$, compared with the NBs + US group.

Abbreviations: NGF, nerve growth factor; US, ultrasound; PLGA, poly(lactic-co-glycolic acid); NBs, nanobubbles; NS, normal saline; ns, not significant.

into cells via sonoporation ${ }^{42}$ and is a desirable physical gene transfection method. US can be used to monitor and crush microbubbles in specific tissues at specific times to achieve the accurate targeting required for gene therapy. Most studies have indicated that, under the right conditions, US does not destroy the exogenous gene but enhances its transfection efficiency. ${ }^{43}$ To date, limited research has been conducted into gene therapy of SCI by US-mediated NB destruction.
Shimamura et $\mathrm{al}^{44}$ intrathecally injected naked luciferase DNA with Optison through the 4/5th lumbar intervertebral space and applied sonication directly on the thoracic dural sac by removing the dorsal part of the 9-10th thoracic vertebra. This approach enhanced the expression of luciferase in the meningeal cells of the insonated region. Takahashi et al ${ }^{17}$ expressed transgenes in meningeal cells by intrathecal injection of luciferase DNA with Optison through the lower lumbar 
A

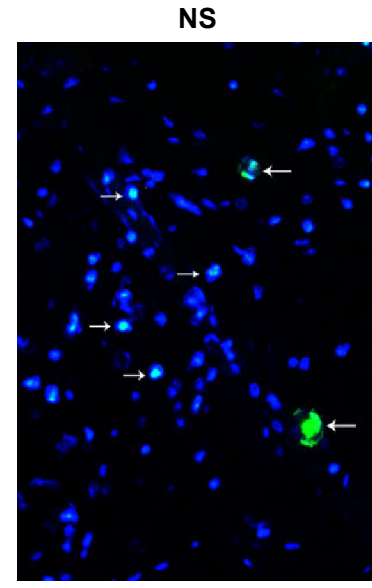

B

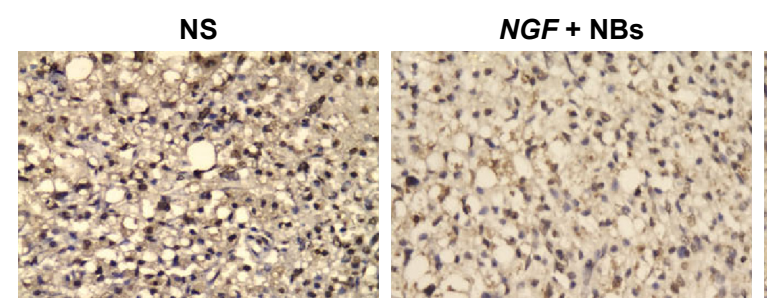

TUNEL-DAPI

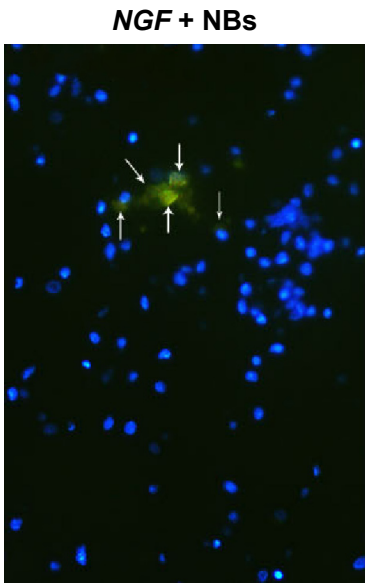

$N G F+$ US
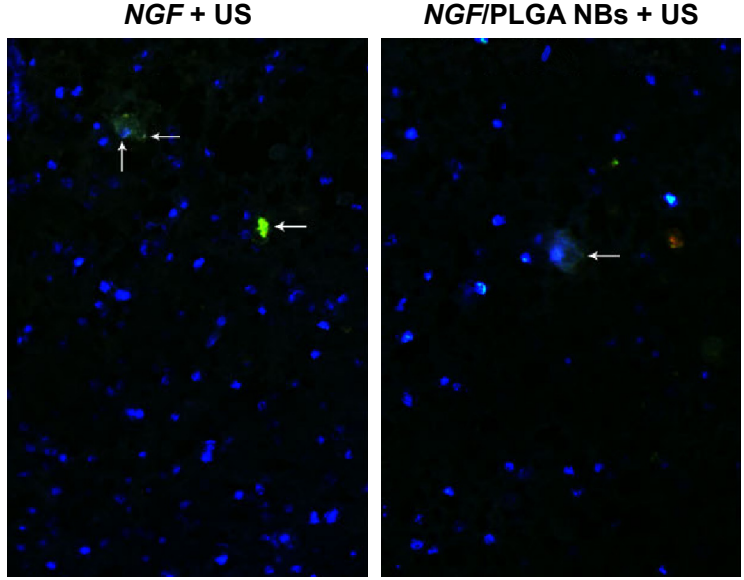

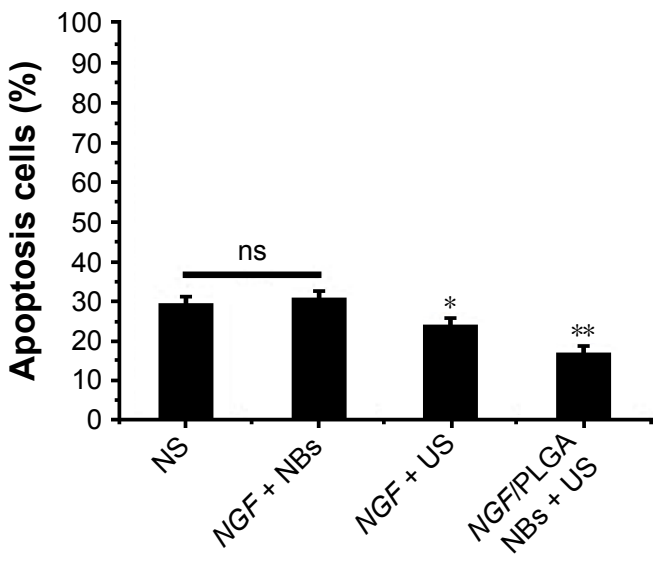

Figure 7 NGF therapy using US-mediated NGF/PLGA NBs destruction inhibits neuronal apoptosis.

Notes: (A) TUNEL-DAPI assay showing reduced apoptosis in the NGF/PLGA NBs + US group. Typical apoptotic cells are indicated by arrows ( $\times 200)$. (B) TUNEL-positive cells in the spinal cord were significantly decreased after NGF therapy through US-mediated NB destruction for I4 days (DAB $\times 400)$. $* P<0.05$, compared with the NS group and $N G F+$ US group. ${ }^{* * P}<0.01$, compared with the other three groups.

Abbreviations: NGF, nerve growth factor; US, ultrasound; PLGA, poly(lactic-co-glycolic acid); NBs, nanobubbles; TUNEL, Terminal deoxynucleotidyl transferase-mediated dUTP-biotin nick end labeling; DAPI, 4',6-diamidino-2-phenylindole; DAB, diaminobenzidine; NS, normal saline; ns, not significant.

intervertebral space, together with transcutaneous insonation at the same lumbar region. With this approach, the target genes adhered to the microbubble and were not wrapped or carried in the microbubble. Thus, the stability of the DNA and Optison mixture is relatively poor. In contrast, we expressed $N G F$ in the spinal cord by direct intravascular injection of $N G F /$ PLGA NBs through the tail vein, together with gene transfection treatment at the SCI region. Our intravenous injection method is more useful clinically. Additionally, the gene transfection instrument (Model UTG 1025, Institution of Ultrasound Imaging, Chongqing Medical University) was developed and patented by our institute. Interestingly, transgenes were consistently expressed for as long as 2 weeks in our study, which is longer than previous reports. Furthermore, previous studies only demonstrated the feasibility of US microbubble-mediated plasmid DNA transfer into the target level of the normal spinal cord and did not examine the injured spinal cords or recovery of neural function.

Foreign genes can be transported into target cells by viral and nonviral vectors. Although viral vectors exhibit 


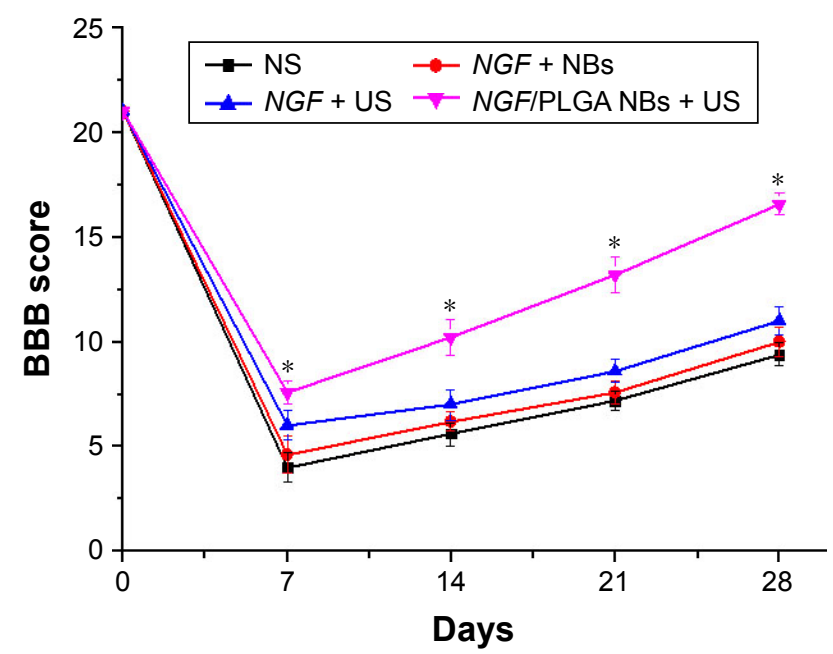

Figure 8 NGF therapy using US-mediated NGF/PLGA NBs destruction improves the recovery of motor function.

Notes: BBB scores of rats in different groups at different time points post-injury. BBB scores for rats that obtained NGF therapy through US-mediated NB destruction rats were significantly higher at $7,14,21$, and 28 days post-injury. ${ }^{*} P<0.05$, compared with other groups.

Abbreviations: NGF, nerve growth factor; US, ultrasound; PLGA, poly(lacticco-glycolic acid); NBs, nanobubbles; BBB, Basso, Beattie, and Bresnahan; NS, normal saline.

a high transfection efficiency, their use is limited by high cytotoxicity and potent immunogenicity. Compared with viral vectors, nonviral vectors such as liposomes and polymeric nanospheres are safe and efficient. ${ }^{11-13}$ PLGA NBs exhibit unique advantages, such as targeting, slow release, and penetrability. ${ }^{14-16}$ These characteristics make PLGA NBs one of the most suitable materials for drug- or gene-based treatment of central nervous system diseases.

In this study, we used PLGA NBs to carry NGF by US into the injured spinal cord to treat SCI. SCI was established in rats using a classical method first reported by Allen. ${ }^{31}$ The histological features and the changes in neural function were similar to those seen in previous studies, indicating successful establishment of our SCI model. ${ }^{45,46}$ NGF and GFP expression in the injured spinal cord was higher in the $N G F /$ PLGA NBs + US group, indicating that NBs can adsorb the target gene plasmids and carry the target gene effectively into the spinal cord in the presence of US irradiation.

Nissl bodies are present on the rough endoplasmic reticulum where proteins are synthesized. They are widely used to reflect the neural structure of brain and spinal cord and are associated with the nutritional condition of neurons. Nissl bodies are reduced in pathological conditions. In this study, Nissl staining was reduced in NS group rats and increased Nissl staining in $N G F /$ PLGA NBs + US rats indicated that $N G F$ therapy through US-mediated NB destruction promoted the regeneration of neurons in the injured spinal cord.
Apoptosis in the spinal cord is a prominent characteristic after SCI. It contributes to the secondary injury processes, and the development of protease inhibitors that prevent programmed cell death is important. ${ }^{47}$ Apoptosis was reduced in rats after $N G F$ therapy through US-mediated NB destruction. This anti-apoptotic effect of NGF is consistent with previous studies and may improve recovery following SCI. ${ }^{48,49}$

Functional recovery is an essential factor for successful SCI treatment. In this study, locomotor function was assessed in rats after SCI. NGF therapy through USmediated NB destruction improved the BBB scores, but there were some complications, which impaired recovery of neurological function. These included death, urinary tract infection, incision infection, and self-destructive behavior. Ten rats died in the four groups. Incisions became infected in four rats and were cured by debridement and suturing. Six rats had urinary tract infections with pyuria, and two rats eventually died of these infections. Overall, seven rats had to be euthanized because of self-destructive behavior. Additionally, a few rats without complications showed poor functional recovery after $N G F /$ PLGA NBs + US treatment. Poor functional recovery manifested as low BBB scores and a long convalescence and may have been caused by intraoperative nerve injury and denervation after surgery. $N G F$ therapy through US-mediated NB destruction significantly improved the recovery of locomotor function recovery in rats. However, our follow-up was only short term, and long-term observation of neural functional recovery must be performed in the future.

There are several limitations that must be considered when interpreting these findings. All rats underwent laminectomy to expose the spinal cord to permit irradiation by US. However, it is difficult for ultrasonic waves to reach the bone-protected spinal cord in patients who do not receive decompression surgery. The development of a new percutaneous transforaminal endoscopic associated with a focused US system will improve future studies of gene therapy in SCI. Furthermore, low-frequency US waves used in this study may have released $N G F$ into other organs, causing supernumerary biological effects. However, despite these limitations, our findings justify further study into the combination of low-frequency focused US and gene therapy associated with microbubbles for SCI treatment.

\section{Conclusion}

We used PLGA NBs to deliver NGF under US irradiation following SCI. This approach improved SCI therapy. Our findings indicate that US-mediated NB destruction facilitates 
gene transfer and may represent a novel gene delivery method for targeted SCI therapy.

\section{Acknowledgments}

This work has been financially supported by National Natural Science Foundation of China (No 81372003). The authors are grateful to Dr Yang Cao for his aid with US imaging and Lan Hao for her help in ultrasonic gene transfection instrument technical support. The authors also acknowledge the Department of Ultrasound Imaging of the Second Affiliated Hospital of Chongqing Medical University for technical assistance.

\section{Disclosure}

The authors report no conflicts of interest in this work.

\section{References}

1. Gomes-Osman J, Cortes M, Guest J, Pascual-Leone A. A systematic review of experimental strategies aimed at improving motor function after acute and chronic spinal cord injury. J Neurotrauma. 2016;33(5): 425-438.

2. Tator $\mathrm{CH}$, Fehlings MG. Review of the secondary injury theory of acute spinal cord trauma with emphasis on vascular mechanisms. JNeurosurg. 1991;75(1):15-26.

3. Yip PK, Malaspina A. Spinal cord trauma and the molecular point of no return. Mol Neurodegener. 2010;7:6.

4. Kattail D, Furlan JC, Fehlings MG. Epidemiology and clinical outcomes of acute spine trauma and spinal cord injury: experience from a specialized spine trauma center in Canada in comparison with a large national registry. J Trauma. 2009;67(5):936-943.

5. Furlan JC, Noonan V, Cadotte DW, Fehlings MG. Timing of decompressive surgery of spinal cord after traumatic spinal cord injury: an evidence-based examination of pre-clinical and clinical studies. J Neurotrauma. 2011;28(8):1371-1399.

6. Ariake K, Ohtsuka H, Motoi F, et al. GCF2/LRRFIP1 promotes colorectal cancer metastasis and liver invasion through integrin-dependent RhoA activation. Cancer Lett. 2012;325(1):99-107.

7. Verghese G, Verma R, Bhutani S. Hyperbaric oxygen therapy in the battlefield. Med J Armed Forces India. 2013;69(1):94-96.

8. Pallini R, Vitiani LR, Bez A, et al. Homologous transplantation of neural stem cells to the injured spinal cord of mice. Neurosurgery. 2005; 57(5):1014-1025.

9. Papale A, Cerovic M, Brambilla R. Viral vector approaches to modify gene expression in the brain. J Neurosci Methods. 2009;185(1):1-14.

10. Hida K, Lai SK, Suk JS, Won SY, Boyle MP, Hanes J. Common gene therapy viral vectors do not efficiently penetrate sputum from cystic fibrosis patients. PLoS One. 2011;6(5):19919.

11. Borden M, Sirsi S, Hernandez S, et al. Polyplex-microbubbles for improved ultrasound-mediated gene therapy. Acoust Soc Am. 2013;133(5): 3409-3409.

12. Mintzer MA, Simanek EE. Nonviral vectors for gene delivery. Chem Rev. 2009;109(2):259-302.

13. Wells DJ. Electroporation and ultrasound enhanced non-viral gene delivery in vitro and in vivo. Cell Biol Toxicol. 2010;26(1):21-28.

14. Feng SS, Mu L, Win KY, et al. Nanoparticles of biodegradable polymers for clinical admini8tration of paclitaxel. Curr Med Chem. 2004; 11(4):413-424.

15. Seiniger SC, Kreuter J, Khalansky AS, et al. Chemotherapy of glioblastoma in rats using doxorubicin-loaded nanoparticles. Int $J$ Cancer. 2004;109(5):759-767.
16. Hynynen K, McDannold N, Vykhodtseva N, et al. Focal disruption of the blood - brain barrier due to $260-\mathrm{kHz}$ ultrasound bursts: a method for molecular imaging and targeted drug delivery. J Neurosurg. 2006;105(3):445-454.

17. Takahashi M, Kido K, Aoi A, Furukawa H, Ono M, Kodama T. Spinal gene transfer using ultrasound and microbubbles. J Control Release. 2007;117(2):267-272.

18. Wang ZG, Ling ZY, Ran HT, et al. Ultrasound-mediated microbubble destruction enhances VEGF gene delivery to the infarcted myocardium in rats. Clin Imaging. 2004;28(6):395-398.

19. Zhong S, Shu S, Wang Z, et al. Enhanced homing of mesenchymal stem cells to the ischemic myocardium by ultrasound-targeted microbubble destruction. Ultrasonics. 2012;52(2):281-286.

20. Xenariou U, Griesenbach U, Liang HD, et al. Use of ultrasound to enhance nonviral lung gene transfer in vivo. Gene Ther. 2007;14(9):768-774.

21. Huang Q, Deng J, Wang F, et al. Targeted gene delivery to the mouse brain by MRI-guided focused ultrasound-induced blood-brain barrier disruption. Exp Neurol. 2012;233(1):350-356.

22. Chen ZY, Liang K, Qiu RX. Targeted gene delivery in tumor xenografts by the combination of ultrasound-targeted microbubble destruction and polyethylenimine to inhibit surviving gene expression and induce apoptosis. J Exp Clin Cancer Res. 2010;29(1):152.

23. Chen S, Shimoda M, Chen J, Matsumoto S, Grayburn PA. Transient overexpression of cyclin D2/CDK4/GLP1 genes induces proliferation and differentiation of adult pancreatic progenitors and mediates islet regeneration. Cell Cycle. 2012;11(4):695-705.

24. Tang HL, Wang ZG, Li Q, et al. Targeted delivery of bone mesenchymal stem cells by ultrasound destruction of microbubbles promotes kidney recovery in acute kidney injury. Ultrasound Med Biol. 2012;38(4):661-669.

25. DuJ, ShiQS, Sun Y, etal.Enhanced delivery ofmonomethoxypoly(ethylene glycol)-poly(lactic-co-glycolicacid)-poly l-lysine nanoparticles loading platelet-derived growth factor BB small interfering RNA by ultrasound and/or microbubbles to rat retinal pigment epithelium cells. J Gene. 2011; 13(6):312-323.

26. Yamaguchi K, Feril LB Jr, Tachibana K, et al. Ultrasound-mediated interferon $\beta$ gene transfection inhibits growth of malignant melanoma. Biochem Biophys Res Commun. 2011;411(1):137-142.

27. Bekeredjian R, Kuecherer HF, Kroll RD, Katus HA, Hardt SE. Ultrasound-targeted microbubble destruction augments protein delivery into testes. Urology. 2007;69(2):386-389.

28. Sofroniew MV, Howe CL, Mobley WC. Nerve growth factor signaling, neuroprotection, and neural repair. Annu Rev Neurosci. 2001;24: 1217-1281.

29. Zhang X, Zheng Y, Wang Z, et al. Methotrexate-loaded PLGA nanobubbles for ultrasound imaging and Synergistic Targeted therapy of residual tumor during HIFU ablation. Biomaterials. 2014;35(19):5148-5161.

30. Ting CY, Fan CH, Liu HL, et al. Concurrent blood-brain barrier opening and local drug delivery using drug-carrying microbubbles and focused ultrasound for brain glioma treatment. Biomaterials. 2012; 33(2):704-712.

31. Allen AR. Surgery of experimental lesions of spinal cord equivalent to crush injury of fracture dislocation - a preliminary report. JAMA. 1911;57:878-880.

32. Livak KJ, Schmittgen TD. Analysis of relative gene expression data using real-time quantitative PCR and the 2(-Delta Delta C(T)) Method. Methods. 2001;25(4):402-408.

33. Basso DM, Beattie MS, Bresnahan JC. A sensitive and reliable locomotor rating scale for open field testing in rats. J Neurotrauma. 1995;12(1):1-21.

34. Andrews MR, Czvitkovich S, Dassie E, et al. Alpha9 integrin promotes neurite outgrowth on tenascin-C and enhances sensory axon regeneration. Neurosci. 2009;29(17):5546-5557.

35. Kwon BK, Liu J, Lam C, et al. Brain-derived neurotrophic factor gene transfer with adeno-associated viral and lentiviral vectors prevents rubrospinal neuronal atrophy and stimulates regeneration associated gene expression after acute cervical spinal cord injury. Spine. 2007;32(11):1164-1173. 
36. Schweitzer JB. Nerve growth factor receptor-mediated transport from CSF labels cholinergic neurons: direct demonstration by a doublelabeling study. Brain Res. 1989;490(2):390-396.

37. Ebendal T. NGF in CNS: experimental data and clinical implications. Prog Growth Factor Res. 1989;1(3):143-159.

38. Hynynen K, McDannold N, Vykhodtseva N, Jolesz FA. Noninvasive MR imaging-guided focal opening of the blood-brain barrier in rabbits. Radiology. 2001;220(3):640-646.

39. Baseri B, Choi JJ, Tung YS, et al. Multi-modality safety assessment of blood-brain barrier opening using focused ultrasound and definity microbubbles: a short-term study. Ultrasound Med Biol. 2010;36(9): 1445-1459.

40. Choi JJ, Selert K, Gao Z, Samiotaki G, Baseri B, Konofagou EE. Noninvasive and localized blood-brain barrier disruption using focused ultrasound can be achieved at short pulse lengths and low pulse repetition frequencies. J Cereb Blood Flow Metab. 2011;31(2):725-737.

41. Kinoshita M, McDannold N, Jolesz FA, Hynynen K. Noninvasive localized delivery of herceptin to the mouse brain by MRI-guided focused ultrasound-induced blood-brain barrier disruption. Proc Natl Acad Sci US A. 2006;103(31):11719-11723.

42. Van Wamel A, Kooiman K, Harteveld M, et al. Vibrating microbubbles poking individual cells: drug transfer into cells via sonoporation. J Control Release. 2006;112(2):149-155.
43. Lu QL, Liang HD, Partridge T, Blomley MJ. Microbubble ultrasound improves the efficiency of gene transduction in skeletal muscle in vivo with reduced tissue damage. Gene Ther. 2003;10(5):396-405.

44. Shimamura M, Sato N, Taniyama Y. Gene transfer into adult rat spinal cord using naked plasmid DNA and ultrasound microbubbles. J Gene Med. 2005;7(11):1468-1474.

45. He B, Nan G. Neuronal regeneration after acute spinal cord injury in adult rats. Spine J. 2016;16(12):1459-1467.

46. Zhang M, Chai Y, Liu T, Xu N, Yang C. Synergistic effects of Buyang Huanwu decoction and embryonic neural stem cell transplantation on the recovery of neurological function in a rat model of spinal cord injury. Exp Ther Med. 2015;9(4):1141-1148.

47. Emery E, Aldana $\mathrm{P}$, Bunge MB, et al. Apoptosis after traumatic human spinal cord injury. J Neurosurg. 1998;89(6):911-920.

48. Liu L, Sun T, Xin F, Cui W, Guo J, Hu J. Nerve growth factor protects against alcohol-induced neurotoxicity in PC12 cells via PI3K/Akt/ mTOR pathway. Alcohol Alcohol. 2017;52(1):12-18.

49. Zhu SP, Wang ZG, Zhao YZ, et al. Gelatin nanostructured lipid carriers incorporating nerve growth factor inhibit endoplasmic reticulum stress-induced apoptosis and improve recovery in spinal cord injury. Mol Neurobiol. 2016;53(7):4375-4386.
International Journal of Nanomedicine

\section{Publish your work in this journal}

The International Journal of Nanomedicine is an international, peerreviewed journal focusing on the application of nanotechnology in diagnostics, therapeutics, and drug delivery systems throughout the biomedical field. This journal is indexed on PubMed Central, MedLine, CAS, SciSearch $®$, Current Contents $\AA /$ Clinical Medicine,

\section{Dovepress}

Journal Citation Reports/Science Edition, EMBase, Scopus and the Elsevier Bibliographic databases. The manuscript management system is completely online and includes a very quick and fair peer-review system, which is all easy to use. Visit http://www.dovepress.com/ testimonials.php to read real quotes from published authors. 